Research Articles

\title{
Study of Tool Wear Properties Using Magnetorheological Fluid Damper
}

\author{
Vikram G Kamble and Vijaykumar Revadi \\ Department of Mechanical Engineering, St Joseph Engineering College, Mangalore, India-575028,
}

Article history

Received: 22-12-2014

Revised: 03-05-2015

Accepted: 15-06-2015

Corresponding Author:

Vikram G Kamble

Mechanical Engineering

Department St Joseph

Engineering College,

Mangalore, India-575028

Email: iknowvikramkamble@gmail.com

\begin{abstract}
Tool wear is one of the important phenomenon in the machining processes. In this article we are explaining the effects of magnetorheological fluids in tool wear process. In our investigation we used the $\mathrm{Al}$ alloy ( $\mathrm{Al}$ 6061) as our test specimen, which has wide range of applications in aerospace industry. We attached our magnetorheological set up to the tool holder to conduct experiments. We conducted different machining processes with and without our magnetorheological fluid setup, after observing the results it was found that the presence of magnetorheological fluid setup during machining processes minimises the tool wear and cutting forces.
\end{abstract}

Keywords: MR Fluid, Damper, Tool Wear, Cutting Force, Surface Roughness, Vibration Amplitude

\section{Introduction}

Tool wear is a very complex phenomenon which can lead to machine down time, product rejects and can also cause problems to personnel (Paul et al., 2015). The magnetorheological fluid acts as a viscoelastic spring with non-linear vibration characteristics that are controlled by parameters like the viscosity index of the fluid medium, shape of the plunger, current through the coil and size of the ferromagnetic particles (Varadarajan et al., 2002). Cutting experiments were conducted to arrive at a set of magnetorheological fluid parameters that can offer better damping characteristics to minimize tool wear and promoting better cutting performance during turning of $6061 \mathrm{Al}$. Conventionally when cylindrical parts requiring high hard-ness as functional requirement are to be machined, the work piece is turned to the near net shape, hardened to the required hardness and ground to the final dimension. This lengthy process cycle can be avoided by hard turning which is intended to replace or limit traditional grinding operations (Byrne et al., 2003). Lord Corporation (LC, 2002) developed magnetorheological fluid shock absorbers for automobiles and it was observed that such shock absorbers can respond instantly and can control varying levels of vibration, shock and motions. Genc and Phule (2002) observed that the properties of the magnetorheological fluids can be varied by varying the parameters associated with magnetorheological fluids like volume, particle size fraction of solids, etc. Also it was reported that using
MR fluid dampers, chatter could be suppressed more effectively by adjusting the damping and natural frequency of the system in boring bar (Mei et al., 2009). Sathianarayanan et al. (2008) investigated the suppression of chatter in boring tools using MR damper and observed that MR damper reduces the possibility of chatter and improves the stability of boring operation. During minimal fluid application, extremely small quantities (about $2 \mathrm{~mL} / \mathrm{min}$ ) of cutting fluids are applied at critical zones in the form of a high velocity pulsed jet so that fluid particles reach the tool chip interface and provide enhanced rake face lubrication. Varadarajan et al. (2002) observed that during minimal fluid application, the overall cutting performance was superior to that during dry turning and conventional wet turning. The life of a tool can come to an end in two major ways. In metal cutting, progressive tool wear occurs in three main forms namely adhesion wear, abrasion wear and diffusion wear. Each wear form has its own characteristic symptoms. Excessive tool vibration is an index of abrasive type of tool wear and it will increase the tool wear and cause poor surface finish during machining (Altintas, 2000). Diffusion and crater type of tool wear are accompanied by excessive cutting temperature and the rate of diffusion increases exponentially with increase in temperature. High cutting force, excessive cutting temperature and increase in tool vibration are indications of progressive tool wear (Paul and Varadarajan, 2012). 
In the present investigation, an attempt was made to develop a magnetorheological fluid damper which can be easily attached to the tool holder for minimising tool vibration and developing cutting performance during hard turning with minimal fluid application.

\section{Development of Magneto-Rheological Fluid Damper}

Magnetorheological (MR) fluids belong to a class of controllable fluids. The essential characteristic of these fluids is their ability to reversibly change from a free-flowing, linear, viscous liquid to a semi-solid with controllable yield strength in milliseconds when exposed to a magnetic field. When exposed to a magnetic field, the suspended particles in the fluid polarize and interact to form a structure aligned with the magnetic field that resists shear deformation or flow. This change in the material appears as a dramatic increase in apparent viscosity, or the fluid develops the characteristics of a semi-solid state. The apparent viscosity and shearing stress can be controlled by changing the intensity of magnetic field (Ginder et al., 1996). The viscosity of MR fluid increases as the strength of the magnetic field increases and even it can behave as semi-solid. When the applied magnetic field vanishes, the MR fluid reverts to its previous, more fluid state. The transformation between the liquid to the semi solid phase takes place very fast i.e. within few milliseconds (Schwartz et al., 2002). A line sketch of the MR Damper developed for this investigation is shown in Fig. 1. It consists of a Plunger (P) made of 410 stainless steel which can moves inside a cup made of stainless steel 410containing MR fluid. MR fluid may be magnetized by passing current through the coil. Threads were cut at the end of the plunger that matched with the threads cut on a hole of the tool holder so that the plunger can be held rigidly with the tool holder as shown in Fig 1. The coil consists of 800 turns of copper wire of 28 gauge. Figure 2 presents a photograph of the location of the MR fluid damper attached to the tool holder and a dissembled view of the MR Fluid Damper is shown in Fig. 3.

When the coil is energized, MR fluid is activated and offers resistance to the motion of the plunger, thereby damping the tool vibration. The damping action of the MR Fluid damper depends on the following factors (i) the Shape (S) of the plunger (ii) the viscosity index (p) of the fluid medium (iii) size (m) of the ferro magnetic particles used for making the MR fluid and (iv) the current (I) through the coil.

\section{Preparation of $\mathrm{Mr}$ Fluid Samples and Experimentation}

Two samples of MR fluids are prepared with carbonyl-iron particles with particle size $2 \mu \mathrm{m}$ to $5 \mu \mathrm{m}$ and density $7830 \mathrm{~kg} / \mathrm{m}^{3}$. The samples are prepared by taking the required quantity of carrier oil and carbonyliron particles. The surfactant used was fine white Lithium grease, the grease was thoroughly mixed with carrier oil for around $2 \mathrm{~h}$ and then the carbonyl-iron particles were added to the mixture and were well stirred for another one h. Hence, we could obtain a homogenous mixture of MR fluid. Preparation of magneto-rheological fluid is explained in article (Vikram, 2014). It was decided to arrive at a set of MR fluid damper parameters namely the shape of the plunger (S), the viscosity index of the fluid medium (p), size of the ferro magnetic particle $(\mathrm{m})$ and the current through the coil(I) to minimize tool vibration. The parameters of MR Fluid damper were as shown in Table 1.

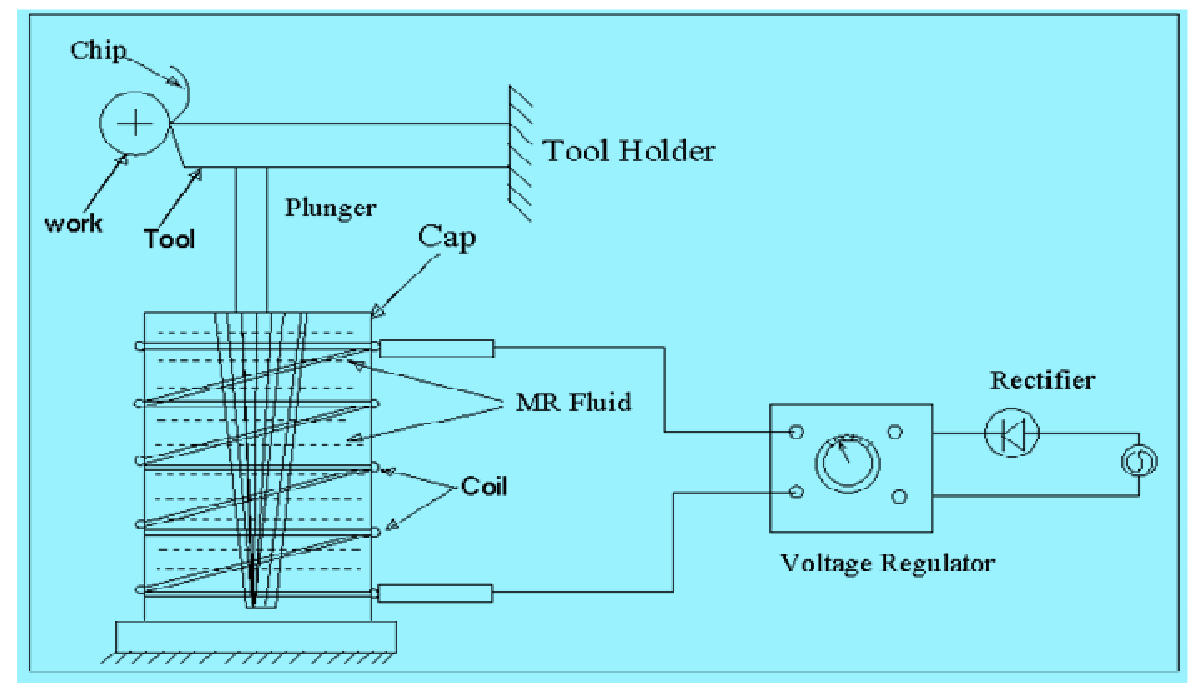

Fig. 1. Line sketch of MR fluid damper 


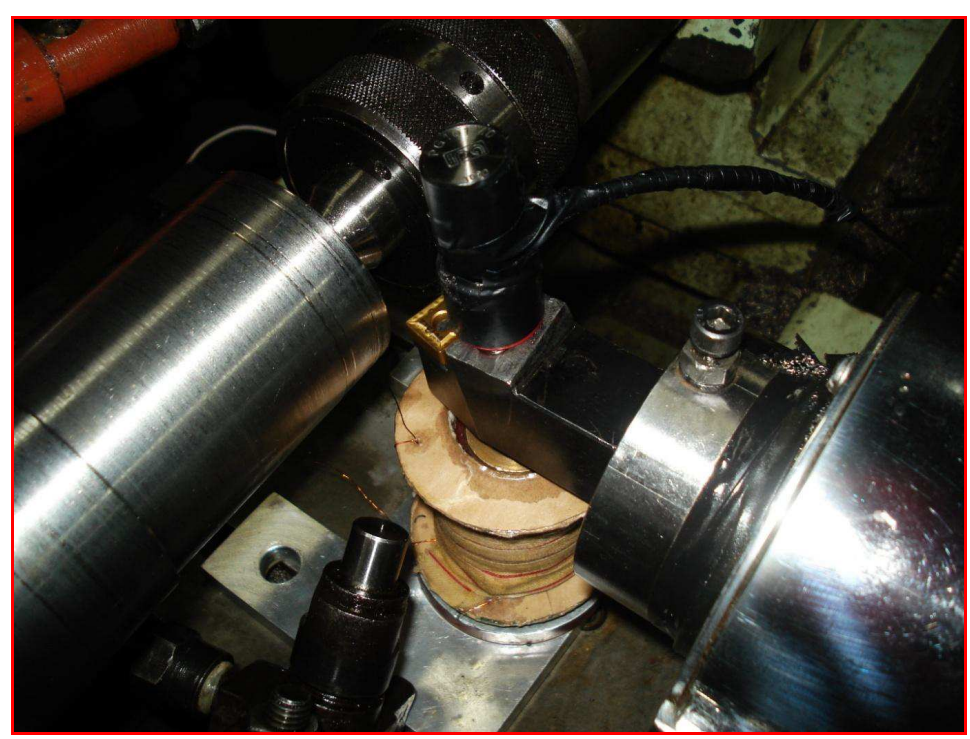

Fig. 2. Photograph of the experimental setup with MR fluid damper

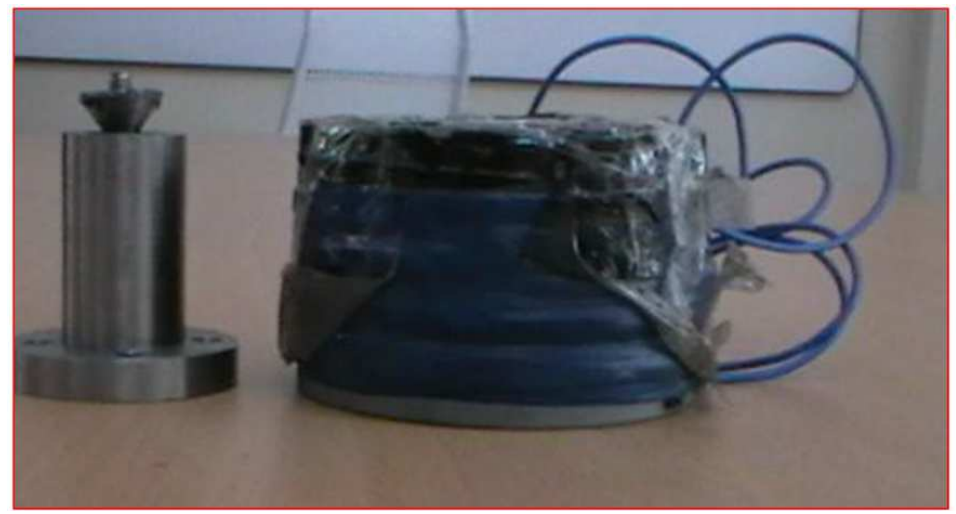

Fig. 3. Dissembled view of the MR Fluid Damper

Table 1. Parameters of MR Fluid damper

\begin{tabular}{ll}
\hline Parameters & \\
\hline Shape of the plunger & Conical \\
Viscosity of the medium[index] & SAE 30 \\
Particle size & $2-5$ microns \\
Current intensity & $20 \mathrm{~V}$ \\
\hline
\end{tabular}

The cutting velocity was kept at $100 \mathrm{~m} / \mathrm{min}$, the feed at $0.12 \mathrm{~mm} / \mathrm{min}$ and the depth of cut at $1.2 \mathrm{~mm}$. The pressure at Fluid applicator was kept at $100 \mathrm{MPa}$, the rate of fluid application at $8 \mathrm{~mL} / \mathrm{min}$, the frequency of pulsing at 500 pulses/min and fluid application was done at tool work interface. Specifications of MR fluid damper is given in the Table 2 .

\section{Experimental Results}

The relative significance of time on tool wear is shown in Fig. 4 and 5 which present the relative significance of the time on cutting force. The relative significance of time on surface roughness and amplitude of tool vibration are shown in Figs. 6 and 7, respectively. The experimental results were analyzed and the levels of input parameters for achieving minimum tool wear, tool vibration, cutting force and surface roughness are shown in Table 3.

From Table 3, it is observed that for obtaining the following results to minimizing tool wear and for achieving better cutting performance, the voltage is maintained at $20 \mathrm{~V}$, direct current is to be used in the coil, a conical shaped plunger is used, the particle size is 2-5 microns and the fluid we used have a viscosity index at SAE30. The strength of the magnetic flux depends on the supply voltage. When the supply voltage is higher, strength of the magnetic field will be high and better will be the damping ability. But an applied voltage at very high magnitude should also be avoided as this may lead to the condition wherein the MR fluid will become a solid mass and loses its damping capability altogether. Moreover higher supply voltage may also result in high temperatures and can lead to safety problems (Paul et al., 2015). 


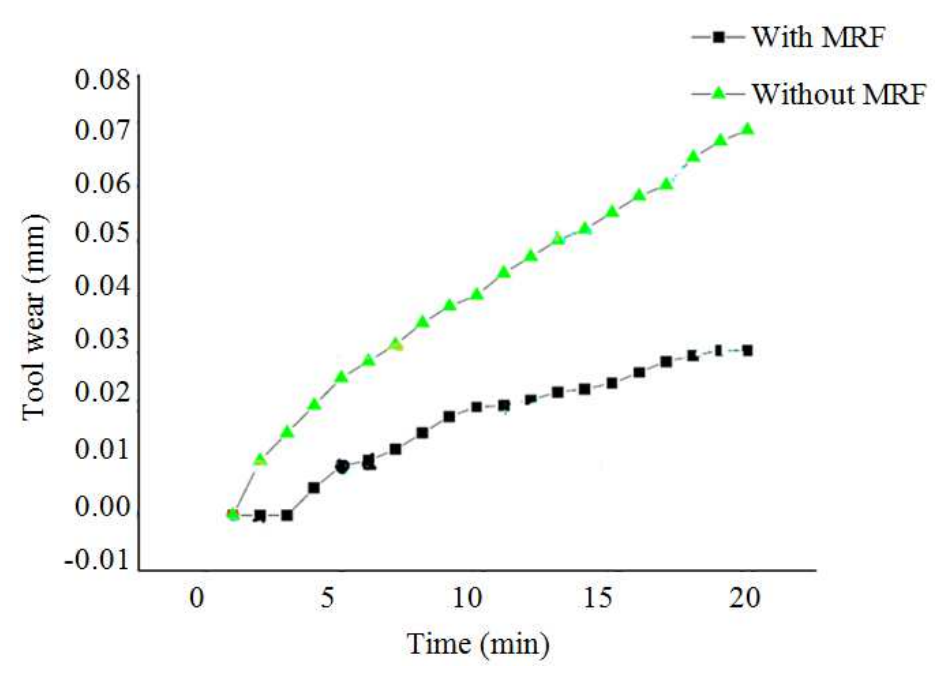

Fig. 4. Relative significance of Time on tool wear

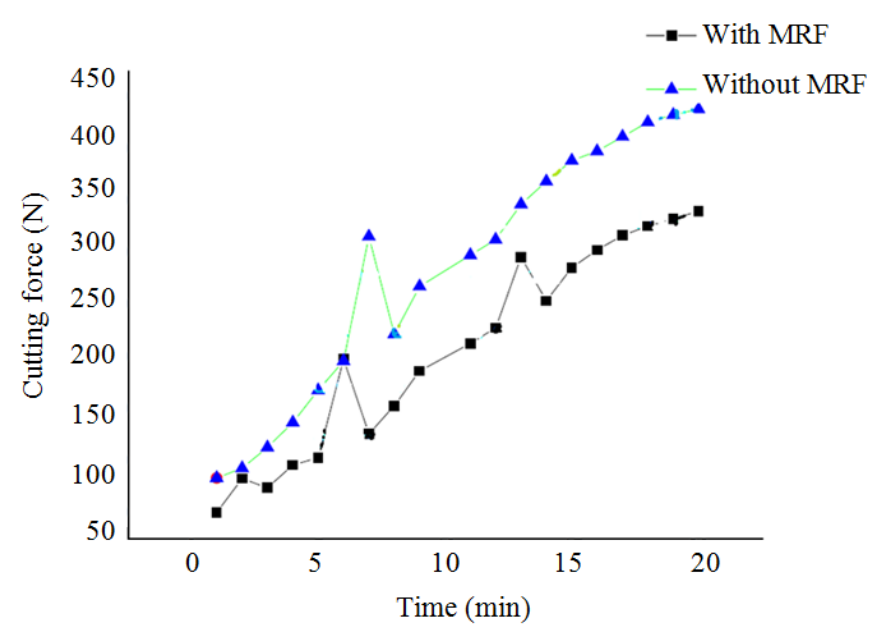

Fig. 5. Relative significance of time on cutting force

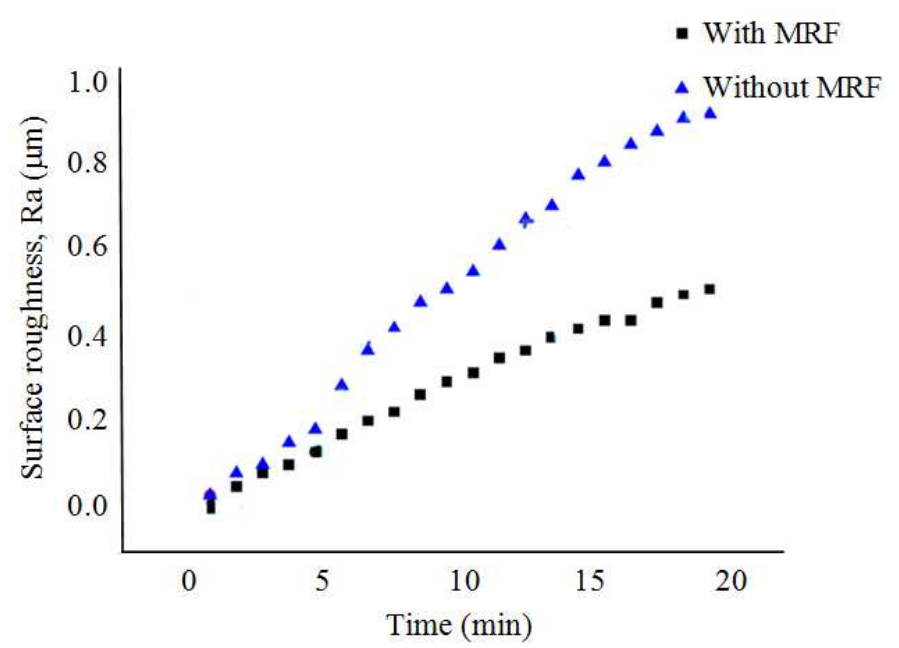

Fig. 6. Relative significance of time on surface roughness 


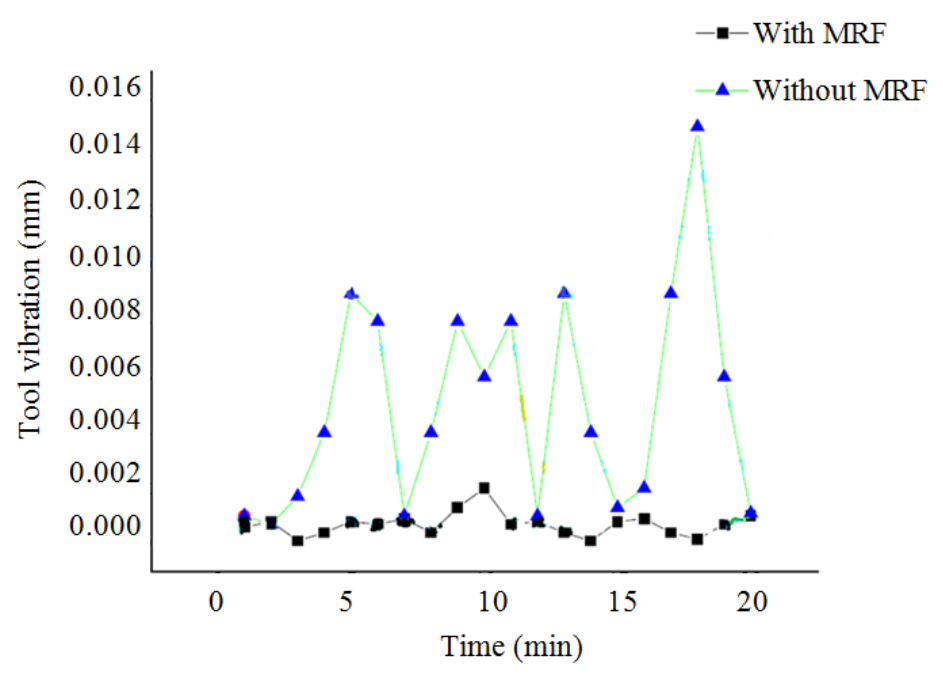

Fig. 7. Relative significance of time on tool vibration

Table 2. Specifications of MR fluid damper

\begin{tabular}{ll}
\hline Size of the plunger & Diameter of plunger $=8 \mathrm{~mm}$ \\
\hline Size of the ferromagnetic particles & $2 \mu \mathrm{m}$ to $5 \mu \mathrm{m}$ \\
Composition of MR fluid & $78: 22: 00(78 \%$ magnetic particles and $22 \%$ oil $)$ \\
Number of turns in the magnetizing coil & 1200 turns \\
Specification of copper wire used in the magnetizing coil & 28 gauge \\
\hline
\end{tabular}

Table 3. Comparison of performance with and without Magnetorheological fluid

\begin{tabular}{lcr}
\hline Parameters & With MR setup & Without MR setup \\
\hline Tool wear, $\mathrm{VB}_{\mathrm{B}}(\mathrm{mm})$ & 0.0250 & 0.0680 \\
Cutting force $(\mathrm{N})$ & 320.8000 & 410.5000 \\
Surface roughness, $\mathrm{Ra}(\mu \mathrm{m})$ & 0.4500 & 0.8900 \\
Amplitude of tool vibration $(\mathrm{mm})$ & 0.0018 & 0.0145 \\
\hline
\end{tabular}

This conical shape helps in better anchoring of the plunger inside the MR fluid. When the plunger has a conical shape, it resists the movement in the downward direction better when compared to any other shapes. A magnetorheological fluid made of oil with viscosity index at SAE 30 provided better cutting performance as this oil can offer higher resistance to the movement of the plunger. Finally it is seen that magnetic particle in the MR fluid should have certain minimum size to offer better performance. If the size of the particles in the MR fluid is too small, there is a possibility of magnetic materials sticking together to form a solid mass when magnetized. But if the size of the particle is sufficiently high (2-5 microns), the tendency to form a solid block reduces. There will be a good distribution of the magnetized particles with the fluid occupying the region in between and this distribution of magnetized particles in a pool of the fluid provides better resistance to the movement of the plunger which leads to reduction in tool wear and improvement in surface finish.

Cutting experiments were conducted with the input parameters kept as indicated in Table 1 and the performance was compared with the cutting performance during conventional minimal fluid application without magnetorheological fluid system. Comparison of the cutting performance is presented in Table 3 .

From the results it was observed that the presence of MR fluid system can suppress the amplitude of tool vibration by $84 \%$, reduce tool wear by $52 \%$, cutting force by $21 \%$ and improve surface finish by $42 \%$ when compared with turning without magnetorheological fluid system (but with minimal fluid application) under the same cutting conditions.

The most prominent component of the cutting force in turning operation is the main cutting force and it acts in the downward direction. The increase in the main cutting force component during hard machining can be considered as a measure of the deterioration of the lubricating ability of the cutting fluid which affects the cutting performance (Paul and Varadarajan, 2013).

Any system that can oppose the movement of the tool in the downward direction can provide better damping. In this investigation, the main cutting force component is reduced effectively due to the presence of magneto rheological fluid which in turn leads to a stable cutting operation with reduced tool wear and improved surface finish. 
In general, the best way to solve a tool vibration problem is to increase the stiffness of the system. When ferro particles of 2-5 micron size mixed with an oil of viscosity index specified by SAE30 and magnetized with direct current provided with a cone type plunger, main cutting force was reduced by $21 \%$. In the case of dry turning, the cutting force was $410 \mathrm{~N}$, with minimal fluid application along with magneto rheological system, the cutting force was as low as $320.8 \mathrm{~N}$. This reduction in cutting force reduces the total energy required to perform cutting operation. The damping force created by the effect of magneto rheological fluid increases the rigidity of the tool which reduces the amplitude of tool vibration by $84 \%$.

The result clearly demonstrates that the presence of magneto rheological fluid can reduce surface roughness effectively. This smooth surface limits the risk of crack initiation (Davim, 2010; 2011) and reduces tool wear effectively through the reduction in cutting force and tool vibration parameters. From the experimental results it was observed that the magneto rheological fluid reduces tool wear by $52 \%$ when compared to a condition in which the tool holder was not provided with a magneto rheological fluid system.

An increase in the cutting length increases the tool wear which, in turn, increases the friction between the tool and the work piece and therefore, the ratio between the thrust force and cutting force increases.

\section{Acknowledgement}

We respectfully thank all staff of Mechanical Engineering Department, St Joseph Engineering College, Mangalore, for their impartial guidance and support throughout our work.

\section{Author's Contributions}

Vikram G Kamble: Planned and conducted the whole Experimental works.

Vijaykumar Revadi: Did mouse work and result analysis.

\section{Ethics}

No ethical issues will arise after the Publication of this work.

\section{References}

Altintas, Y., 2000. Manufacturing Automation: Metal Cutting Mechanics, Machine Tool Vibrations and CNC Design. 1st Edn., Cambridge University Press, UK.

Byrne, G., D. Dornfeld and B. Denkena, 2003. Advancing cutting technology. CIRP AnnalsManufac. Technol., 52: 483-507.

DOI: $10.1016 / \mathrm{S} 0007-8506(07) 60200-5$
Davim, J.P., 2010. Surface integrity in machining. 1st Edn., Springer Science and Business Media, London ISBN-10: 1848828748, pp: 215.

Davim, J.P., 2011. Machining of hard materials. Springer Science and Business Media, London, ISBN-10: 1849964505, pp: 211.

Ginder, J.M., L.C. Davis and L.D. Elie, 1996. Rheology of magnetorheological fluids: Models and measurements. Int. J. Mod. Phys. B, 10: 3293-3303. DOI: $10.1142 / \mathrm{S} 0217979296001744$

Genc, S. and P.P. Phule, 2002. Rheological properties of magnetorheological fluids. Smart Materials Structures 11: 140-14. DOI: 10.1088/0964-1726/11/1/316

LC, 2002. Lord Corporation.

Mei, D., T. Kong, A.J. Shih and Z. Chen, 2009. Magnetorheological fluid-controlled boring bar for chatter suppression. J. Materials Processing Technol., 209: 1861-1870.

DOI: 10.1016/j.jmatprotec.2008.04.037

Paul, P.S. and A.S. Varadarajan, 2012. A multi-sensor fusion model based on artificial neural network to predict tool wear during hard turning. J. Eng. Manufacture, 5: 853-860. DOI: $10.1177 / 0954405411432381$

Paul, P.S. and A.S. Varadarajan, 2013. Performance evaluation of hard turning of AISI 4340 steel with minimal fluid application in the presence semi solid lubricants. J. Eng. Tribol., DOI: $10.1177 / 1350650112468376$

Paul, P.S., A.S. Varadarajan and S. Mohanasundaram, 2015. Effect of magnetorheological fluid on tool wear during hard turning with minimal fluid application. Archives Civil Mechanical Eng., 15: 124-132. DOI: 10.1016/j.acme.2014.03.007

Sathianarayanan, D., L. Karunamoorthy, J. Srinivasan, G.S. Kandasami and K. Palanikumar, 2008. Chatter suppression in boring operation using magnetorheological fluid damper. Materials Manufacturing Processes, 23: 329-335. DOI: $10.1080 / 10426910701860897$

Schwartz, B., A. Ward, J. Monterosso, S. Lyubomirsky and $\mathrm{K}$. White et al., 2002. Maximizing versus satisficing: Happiness is a matter of choice. J. Pers Soc. Psychol., 83: 1178-97. PMID: 12416921

Varadarajan, A.S., P.K. Philip and B. Ramamoorthy, 2002. Investigations on Hard Turning with Minimal cutting Fluid application (HTMF) and its comparison with dry and wet turning. Int. J. Machine Tools Manufacture, 42: 193-200.

DOI: 10.1016/S0890-6955(01)00119-5

Vikram, K.., et al., 2014. Am. J. Nanotechnol., 5: 12-16. 